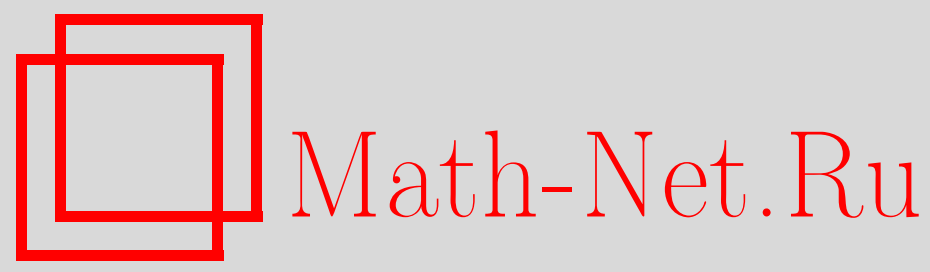

Р. В. Константинов, О квазилинейной дифференциальной игре преследования с простой динамикой при наличии фазового ограничения, Матем. заметки, 2001, том 69, выпуск 4, 581-590

DOI: https://doi.org/10.4213/mzm524

Использование Общероссийского математического портала Math-Net.Ru подразумевает, что вы прочитали и согласны с пользовательским соглашением http://www . mathnet.ru/rus/agreement

Параметры загрузки:

IP : 52.87 .193 .239

26 апреля 2023 г., 13:30:30

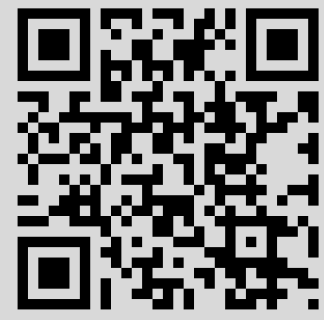




\section{О КВАЗИЛИНЕЙНОЙ ДИФФЕРЕНЦИАЛЬНОЙ ИГРЕ ПРЕСЛЕДОВАНИЯ С ПРОСТОЙ ДИНАМИКОЙ ПРИ НАЛИЧИИ ФАЗОВОГО ОГРАНИЧЕНИЯ}

\section{P.В. Константинов}

В работе рассматривается квазилинейная дифференциальная игра преследования на фиксированном отрезке времени с выпукльм компактным целевым множеством при наличии фазового ограничения - выпуклого замкнутого множества. В работе построено выпуклое компактное множество гарантированной поимки, аналогичное альтернированной сумме Понтрягина, и определена гарантированная кусочно-программная стратегия преследователя, обеспечивающая попадание фазового вектора в конечный момент времени на целевое множество, причем в течении игры значение фазового вектора удовлетворяет фазовому ограничению. При определенных условиях доказана сходимость построенной альтернированной суммы в метрике Хаусдорфа к выпуклому компактному множеству - аналогу альтернированного интеграла Понтрягина для рассматриваемой дифференциальной игры.

Библиография: 5 названий.

1. Постановка задачи. Рассматривается управляемая система [1], [2]

$$
\frac{d z}{d t}=f(u, v), \quad u \in P, \quad v \in Q
$$

где $z \in \mathbb{R}^{n}$ - фазовый вектор, $t \in[0, T]$ - время, $u$ и $v$ - управляющие параметры. Множества $P \subset \mathbb{R}^{p}$ и $Q \subset \mathbb{R}^{q}$ являются компактами. Функция $f: P \times Q \rightarrow \mathbb{R}^{n}$ непрерывна. Задано начальное значение фазового вектора $z(0)=z_{0}$. Допустимыми управлениямu игроков будем называть кусочно-непрерьвные на $[0, T]$ функции $u(\cdot)$ и $v(\cdot)$ со значениями в $P$ и $Q$ соответственно. Задано вьпуклое компактное множество $M \subset \mathbb{R}^{n}$ и выпуклое замкнутое множество $R \subset \mathbb{R}^{n}$.

Первьй игрок (преследователь) имеет в своем распоряжении управляющий параметр $u$. Управляюший параметр $v$ является помехой, его значениями распоряжается второй игрок (убегающий). Целью первого игрока является выполнение включений

$$
z(T) \in M, \quad z(t) \in R \text { для всех } t \in[0, T] .
$$

Для решения поставленной игровой задачи преследователь использует следующую кусочно-программную стратегию. До начала игры он выбирает разбиение $\omega=\left\{t_{k}\right\}_{k=0}^{K}$ отрезка $[0, T]$, т.е. $0=t_{0}<t_{1}<\cdots<t_{K}=T$. Преследователь не имеет никакой

Работа выполнена при финансовой поддержке Российского фонда фундаментальных исследований, грант № 98-01-00645, и Конкурсного центра фундаментального естествознания, грант № 97-0-1.9-26. 
информации о реализующихся в ходе игры значениях управляющего параметра убегающего. Параметры игровой задачи (множества $P, Q, R, M$; функция $f$; момент окончания игры $T$ ) известны преследователю. В каждый момент времени $t_{k}, k \in \overline{0, K-1}$, преследователь строит кусочно-постоянное управление $u_{k}(\cdot)$ на интервал $\left[t_{k}, t_{k+1}\right)$ по информации о реализовавшемся значении $z_{k}=z\left(t_{k}\right)$.

2. Альтернированные множества. Напомним, что суммой и геометрической разностью множеств $A \subset \mathbb{R}^{n}$ и $B \subset \mathbb{R}^{n}$ называются соответственно множества (см. [3])

$$
A+B=\{a+b: a \in A, b \in B\}, \quad A * B=\left\{c \in \mathbb{R}^{n}: c+B \subset A\right\} .
$$

Выпуклую оболочку множества $A$ (см. $[4$, с. 9]) обозначим со $A$. Замыкание множества $A$ (см. [4, с. 11]) обозначим $\mathrm{cl} A$. Полунорму множества $A$ (см. [5, с. 13]) обозначим $\|A\|=\sup \{|a|: a \in A\}$. Шар в $\mathbb{R}^{n}$ радиуса $r$ с центром в нуле обозначим $\mathbb{B}_{r}=\left\{z \in \mathbb{R}^{n}\right.$ : $|z| \leqslant r\}$.

Пусть $\mathscr{K}$ - пространство вьпуклых компактных подмножеств $\mathbb{R}^{n}$ с хаусдорфовой метрикой $h(\cdot, \cdot)$ (см. [5. с. 11]). Пусть многозначное отображение $F: P \rightarrow \mathscr{K}$ таково, что $F(u)=\cos f(u, Q)$ для любого $u \in P$. Пусть число $r=\|f(P, Q)\|$. Для любого разбиения $\omega=\left\{t_{k}\right\}_{k=0}^{K}$ отрезка $[0, T]$ определяем множества: $M_{K}(\omega)=M$,

$$
M_{k}(\omega)=\operatorname{co} \bigcup_{u \in P}\left[\left(M_{k+1}(\omega) * F(u) \Delta t_{k}\right) \cap\left(R * \mathbb{B}_{r} \Delta t_{k}\right)\right],
$$

где $\Delta t_{k}=t_{k+1}-t_{k}, k \in \overline{0, K-1}$.

Множество $M_{0}(\omega)$ является аналогом альтернированной суммы (см. [3]) для рассматриваемой квазилинейной дифференциальной игры преследования.

ТЕорема 1. Для любого разбиения $\omega$ отрезка $[0, T]$ множество $M_{0}(\omega)$ является выпуклым компактом в $\mathbb{R}^{n}$ (быть может, пустым), причем

$$
M_{0}(\omega) \subset(\|M\|+r T) \mathbb{B}_{1}
$$

Доказательство теоремы 1 опирается на следующие леммы.

Лемма 1. Многозначное отображение $F$ непрерывно в $P$.

ДокАЗАТЕльство. В силу непрерьвности функции $f$ на компакте $P \times Q$ она является равномерно непрерывной на нем. Следовательно, для любого $\varepsilon>0$ существует $\delta>0$ такое, что для всех $u_{1}, u_{2} \in P$ и $v_{1}, v_{2} \in Q$ таких, что $\left|u_{1}-u_{2}\right|<\delta,\left|v_{1}-v_{2}\right|<\delta$, выполнено условие

$$
f\left(u_{1}, v_{1}\right) \in f\left(u_{2}, v_{2}\right)+\mathbb{B}_{\varepsilon} \subset F\left(u_{2}\right)+\mathbb{B}_{\varepsilon} .
$$

Так как $v_{1} \in Q$ произвольно, то $f\left(u_{1}, Q\right) \subset F\left(u_{2}\right)+\mathbb{B}_{\varepsilon}$. Так как правая часть последнего включения выпукла, то

$$
F\left(u_{1}\right) \subset F\left(u_{2}\right)+\mathbb{B}_{\varepsilon}
$$

Аналогично находим, что

$$
F\left(u_{2}\right) \subset F\left(u_{1}\right)+\mathbb{B}_{\varepsilon} .
$$

Следовательно, $h\left(F\left(u_{1}\right), F\left(u_{2}\right)\right) \leqslant \varepsilon$ для всех $u_{1}, u_{2} \in P$ таких, что $\left|u_{1}-u_{2}\right|<\delta$. Лемма доказана. 
Лемма 2. Пусть $K \in \mathscr{K}$, иисло $\delta>0$. Пусть

$$
K_{1}=\bigcup_{u \in P}\left[(K \stackrel{*}{*} F(u) \delta) \cap\left(R * \mathbb{B}_{r} \delta\right)\right], \quad K_{2}=\operatorname{co} K_{1} .
$$

Тогда $K_{1}$ и $K_{2}$ являются компактами в $\mathbb{R}^{n}$.

ДоКАЗАТЕЛЬСтво. Компактность $K_{1}$ влечет компактность $K_{2}$ в силу теоремы 1.7 [ 4 , c. 16]. Множество $K_{1}$ ограничено, так как $K_{1} \subset(\|K\|+r \delta) \mathbb{B}_{1}$. Покажем замкнутость $K_{1}$. Пусть последовательность $\left\{z_{m}\right\} \subset K_{1}$ такова, что $z_{m} \rightarrow z_{0}$ при $m \rightarrow \infty$. Для любого номера $m$ существует $u_{m} \in P$ такое, что

$$
z_{m} \in\left(K \stackrel{*}{*} F\left(u_{m}\right) \delta\right) \cap\left(R \stackrel{*}{*} \mathbb{B}_{r} \delta\right) .
$$

В силу компактности $P$, переходя, если нужно, к подпоследовательности, будем считать, что $u_{m} \rightarrow u_{0}$ при $m \rightarrow \infty$. Следовательно, для любого номера $m$ вьполнены включения

$$
z_{m}+F\left(u_{m}\right) \delta \subset K, \quad z_{m}+\mathbb{B}_{r} \delta \subset R .
$$

Переходя к пределу при $m \rightarrow \infty$, пользуясь непрерывностью $F$ в точке $u_{0} \in P$, доказанной в лемме 1 , и замкнутостью множеств $K$ и $R$, получаем

$$
z_{0}+F\left(u_{0}\right) \delta \subset K, \quad z_{0}+\mathbb{B}_{r} \delta \subset R .
$$

Таким образом, $z_{0} \in K_{1}$. Лемма доказана.

ДокаЗАТЕЛЬСТво теоремЫ 1 . Пусть $\omega=\left\{t_{k}\right\}_{k=0}^{K}$ - разбиение отрезка $[0, T]$. Выпуклость множеств $M_{k}(\omega)$ для всех $k \in \overline{0, K}$ следует непосредственно из их определения. Докажем, что для любого $k \in \overline{0, K}$ выполнено включение

$$
M_{k}(\omega) \subset\left(\|M\|+r\left(T-t_{k}\right)\right) \mathbb{B}_{1} .
$$

Действительно, для $k=K$ включение вьполнено, так как $M_{K}(\omega)=M \subset\|M\| \mathbb{B}_{1}$. Если оно справедливо для $k+1$, где $k \in \overline{0, K-1}$, то $\left\|M_{k+1}(\omega)\right\| \leqslant\|M\|+r\left(T-t_{k+1}\right)$. Следовательно,

$$
M_{k}(\omega) \subset\left(\left\|M_{k+1}(\omega)\right\|+r \Delta t_{k}\right) \mathbb{B}_{1} \subset\left(\|M\|+r\left(T-t_{k}\right)\right) \mathbb{B}_{1} .
$$

Таким образом, включение (4) по индукции доказано. Множество $M_{K}(\omega)=M$ является выпуклым компактом. Если $M_{k+1}(\omega)$, где $k \in \overline{0, K-1}$, является вьпуклым компактом, то по лемме 2 таковым является и множество $M_{k}(\omega)$. Таким образом, по индукции доказано, что множество $M_{0}(\omega)$ является вьпуклым компактом. Теорема доказана.

Определим множество

$$
\widetilde{M}=\operatorname{cl} \bigcup_{\omega} M_{0}(\omega)
$$

где объединение берется по всевозможньм разбиениям $\omega$ отрезка $[0, T]$. В силу теоремы 1 множество $\widetilde{M}$ компактно (быть может, пусто) в $\mathbb{R}^{n}$. Множество $\widetilde{M}$ является аналогом альтернированного интеграла Понтрягина (см. [3]) для рассматриваемой квазилинейной дифференциальной игры преследования. 
ТЕОРема 2. Пусть $z_{0}=z(0)$ является внутренней точкой множества $\widetilde{M}$ (см. [4, с. 11]). Тогда существует кусочно-программная стратегия преследователя, гарантирующая при любом допустимом поведении убегающего успешное окончание игры преследования в момент $T$.

ДокАЗАТЕЛьство. Так как $z_{0}$ является внутренней точкой множества $\widetilde{M}$, то в силу определения $\widetilde{M}$ существует разбиение $\omega=\left\{t_{k}\right\}_{k=0}^{K}$ отрезка $[0, T]$ такое, что $z_{0} \in M_{0}(\omega)$.

Предположим, рассуждая по индукции, что для номера $k \in \overline{0, K-1}$ вьполнено включение $z_{k}=z\left(t_{k}\right) \in M_{k}(\omega)$. Тогда по теореме Каратеодори (см. [4, с. 9]) существует натуральное число $m \leqslant n+1$, положительные числа $\left\{\alpha_{i}\right\}_{i=1}^{m}, \sum_{i=1}^{m} \alpha_{i}=1$, векторы

$$
x_{i} \in \bigcup_{u \in P}\left[\left(M_{k+1}(\omega) \stackrel{*}{*} F(u) \Delta t_{k}\right) \cap\left(R \stackrel{*}{*} B_{r} \Delta t_{k}\right)\right]
$$

такие, что вьполнено равенство $z_{k}=\sum_{i=1}^{m} \alpha_{i} x_{i}$. Следовательно, для каждого $i \in \overline{1, m}$ существует вектор $u_{i} \in P$ такой, что

$$
x_{i}+F\left(u_{i}\right) \Delta t_{k} \subset M_{k+1}(\omega), \quad x_{i}+\mathbb{B}_{r} \Delta t_{k} \subset R .
$$

Последнее включение в силу вьпуклости множества $R$ означает, что

$$
z_{k}+\mathbb{B}_{r} \Delta t_{k} \subset R
$$

т.е. для любых допустимых управлений $u(\cdot), v(\cdot)$, определенных на интервале $\left[t_{k}, t_{k+1}\right)$, справедливо включение

$$
z(t)=z_{k}+\int_{t_{k}}^{t} f(u(\tau), v(\tau)) d \tau \in R
$$

для всех $t \in\left[t_{k}, t_{k+1}\right]$.

Определим кусочно-постоянное управление преследователя $u_{k}(\cdot)$ на $\left[t_{k}, t_{k+1}\right)$ следуюшим образом. Пусть

$$
\tau_{0}=t_{k}, \quad \tau_{s}=t_{k}+\sum_{i=1}^{s} \alpha_{i} \Delta t_{k}, \quad s \in \overline{1, m} .
$$

Тогда определяем $u_{k}(t)=u_{s}$ для любого $t \in\left[\tau_{s-1}, \tau_{s}\right)$, где $s \in \overline{1, m}$. Следовательно, для любого допустимого управления убегающего $v(\cdot)$, определенного на интервале $\left[t_{k}, t_{k+1}\right)$, справедливо включение

$$
z_{k}+\int_{t_{k}}^{t_{k+1}} f\left(u_{k}(t), v(t)\right) d t \in \sum_{i=1}^{m} \alpha_{i}\left(x_{i}+F\left(u_{i}\right) \Delta t_{k}\right) \subset M_{k+1}(\omega) .
$$

Таким образом, выбор преследователем управления $u_{k}(\cdot)$ в момент $t_{k}$ гарантирует выполнение включений

$$
z\left(t_{k+1}\right) \in M_{k+1}(\omega), \quad z(t) \in R \quad \forall t \in\left[t_{k}, t_{k+1}\right] .
$$

Следовательно, по индукции доказали существование кусочно-программной стратегии преследователя, гарантирующей вьполнение включений (2). Теорема доказана. 
3. Монотонность альтернированных множеств. При измельчении разбиения $\omega$ альтернированная сумма $M_{0}(\omega)$ не убывает по включению, т.е. справедлива

Теорема 3. Пусть $\omega_{1} u \omega_{2}-$ два разбиения отрезка $[0, T]$, причем $\omega_{1} \prec \omega_{2}$. Тогда $M_{0}\left(\omega_{1}\right) \subset M_{0}\left(\omega_{2}\right)$.

Доказательство теоремы 3 опирается на следующую лемму.

ЛЕмма 3. Пусть $K_{0} \in \mathscr{K}, \delta_{1}>0, \delta_{2}>0, \delta_{3}=\delta_{1}+\delta_{2}$. Пусть

$$
\begin{aligned}
& K_{1}=\operatorname{co} \bigcup_{u \in P}\left[\left(K_{0} * * F(u) \delta_{1}\right) \cap\left(R * \mathbb{B}_{r} \delta_{1}\right)\right], \\
& K_{2}=\operatorname{co} \bigcup_{u \in P}\left[\left(K_{1} * F(u) \delta_{2}\right) \cap\left(R * \mathbb{B}_{r} \delta_{2}\right)\right], \\
& K_{3}=\operatorname{co} \bigcup_{u \in P}\left[\left(K_{0} * * F(u) \delta_{3}\right) \cap\left(R * \mathbb{B}_{r} \delta_{3}\right)\right] .
\end{aligned}
$$

Тогда $K_{3} \subset K_{2}$.

ДокАЗАТЕЛЬСТВо. Пусть $\tilde{u} \in P$. Используя свойства вьпуклой оболочки и геометрической разности множеств (см. [5, с. 11]), находим

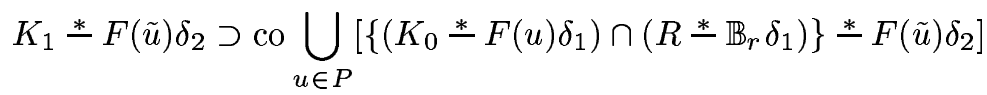

$$
\begin{aligned}
& \supset \operatorname{co} \bigcup_{u \in P}\left[\left(K_{0} \stackrel{*}{*}\left(F(u) \delta_{1}+F(\tilde{u}) \delta_{2}\right)\right) \cap\left(R \stackrel{*}{\mathbb{B}_{r}} \delta_{3}\right)\right] \text {. }
\end{aligned}
$$

Так как $R * \mathbb{B}_{r} \delta_{2} \supset R * \mathbb{B}_{r} \delta_{3}$, то получаем включение

$$
\left(K_{1} * F(\tilde{u}) \delta_{2}\right) \cap\left(R * \mathbb{B}_{r} \delta_{2}\right) \supset \operatorname{co} \bigcup_{u \in P}\left[\left(K_{0} *\left(F(u) \delta_{1}+F(\tilde{u}) \delta_{2}\right)\right) \cap\left(R * \mathbb{B}_{r} \delta_{3}\right)\right]
$$

Тогда в силу произвольности вектора $\tilde{u} \in P$ получаем

$$
\begin{aligned}
K_{2} & =\operatorname{co} \bigcup_{\tilde{u} \in P}\left[\left(K_{1} * F(\tilde{u}) \delta_{2}\right) \cap\left(R * \mathbb{B}_{r} \delta_{2}\right)\right] \\
& \supset \operatorname{co} \bigcup_{\tilde{u} \in P} \operatorname{co} \bigcup_{u \in P}\left[\left(K_{0} *\left(F(u) \delta_{1}+F(\tilde{u}) \delta_{2}\right)\right) \cap\left(R * \mathbb{B}_{r} \delta_{3}\right)\right] .
\end{aligned}
$$

Последнее множество содержит в качестве подмножества

$$
\operatorname{co} \bigcup_{u \in P}\left[\left(K_{0} *\left(F(u) \delta_{1}+F(u) \delta_{2}\right)\right) \cap\left(R * \mathbb{B}_{r} \delta_{3}\right)\right]=K_{3},
$$

т.е. $K_{2} \supset K_{3}$. Лемма доказана. 
ДОКАЗАТЕЛЬСТВО ТЕОРЕМЫ 3. Пусть разбиения отрезка $[0, T]$ имеют следующий вид: $\omega_{1}=\left\{t_{k}\right\}_{k=0}^{K}, \omega_{2}=\left\{\tau_{s}\right\}_{s=0}^{S}$. Так как вьполнено условие $\omega_{1} \prec \omega_{2}$, то существуют номера $\left\{s_{k}\right\}_{k=0}^{K}$ такие, что $0=s_{0}<s_{1}<\cdots<s_{K}=S, \tau_{s_{k}}=t_{k}$ для всех $k \in \overline{0, K}$.

По определению $M=M_{s_{K}}\left(\omega_{2}\right)=M_{K}\left(\omega_{1}\right)$. Предположим, рассуждая по индукции, что для $k \in \overline{1, K}$ вьполнено включение $M_{s_{k}}\left(\omega_{2}\right) \supset M_{k}\left(\omega_{1}\right)$. Тогда в силу леммы 3 получаем

$$
\begin{aligned}
M_{s_{k-1}}\left(\omega_{2}\right) & \supset \operatorname{co} \bigcup_{u \in P}\left[\left(M_{s_{k}}\left(\omega_{2}\right) * F(u) \Delta t_{k-1}\right) \cap\left(R * \mathbb{B}_{r} \Delta t_{k-1}\right)\right] \\
& \supset \operatorname{co} \bigcup_{u \in P}\left[\left(M_{k}\left(\omega_{1}\right) * F(u) \Delta t_{k-1}\right) \cap\left(R * \mathbb{B}_{r} \Delta t_{k-1}\right)\right]=M_{k-1}\left(\omega_{1}\right) .
\end{aligned}
$$

Таким образом, по индукции доказано включение $M_{0}\left(\omega_{2}\right) \supset M_{0}\left(\omega_{1}\right)$. Теорема доказана.

4. Сходимость альтернированных множеств. Исследуем поведение альтернированной суммы $M_{0}(\omega)$, если мелкость разбиения $|\omega| \rightarrow 0$. Для любого разбиения $\omega=\left\{t_{k}\right\}_{k=0}^{K}$ отрезка $[0, T]$ и вектора $u \in P$ определим для удобства множества

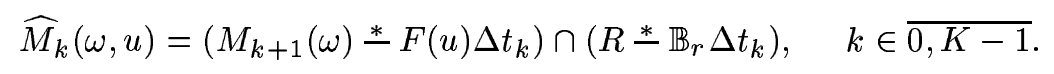

ТЕорема 4. Пусть существуют $r_{0}>0 u \delta_{0}>0$ такие, что для любого разбиения $\omega=\left\{t_{k}\right\}_{k=0}^{K}$ отрезка $[0, T]$ мелкости $|\omega|<\delta_{0}$ выполнено условие

$$
\widehat{M}_{k}(\omega, u) \stackrel{*}{\mathbb{B}_{r_{0}}} \neq \varnothing
$$

для всех $u \in P, k \in \overline{0, K-1}$. Тогда

$$
\lim _{|\omega| \rightarrow 0} h\left(M_{0}(\omega), \widetilde{M}\right)=0
$$

Доказательство теоремы 4 опирается на следующие леммы.

ЛЕмма 4. Пусть $\mathbb{E}$ - конечномерное нормированное пространство с нормой $|\cdot| \mathbb{E}$. Пусть $\Omega-$ подмно сество $\mathbb{E}$. Пусть многозначное отобрахсение $G_{0}: \Omega \times P$ $\rightarrow \mathscr{K}$ является полунепрерывным сверху в точке $\omega_{0} \in \Omega$ (см. [5, с. 19]) равномерно по $u \in P$. Пусть многозначные отображсени $G_{1}: \Omega \rightarrow \mathscr{K} u G_{2}: \Omega \rightarrow \mathscr{K}$ являются полунепрерывными снизу в точке $\omega_{0} \in \Omega$ (см. [5, с. 19]). Пусть многозначные отображения $H_{1}: \Omega \times P \rightarrow \mathscr{K}$ и $H_{2}: \Omega \times P \rightarrow \mathscr{K}$ таковы, что

$$
H_{1}(\omega, u)=G_{1}(\omega) \stackrel{*}{-} G_{0}(\omega, u), \quad H_{2}(\omega, u)=H_{1}(\omega, u) \cap G_{2}(\omega)
$$

для всех $\omega \in \Omega, u \in P$. Пусть существуют числа $r_{1}>0 u r_{2}>0$ такие, что $H_{1}\left(\omega_{0}, u\right) \subset \mathbb{B}_{r_{1}}, H_{2}\left(\omega_{0}, u\right) \stackrel{*}{\mathbb{B}_{r_{2}}} \neq \varnothing$ для всех $u \in P$. Тогда многозначные отображения $\mathrm{H}_{1}$ и $\mathrm{H}_{2}$ являются полунепрерывными снизу в точке $\omega_{0} \subset \Omega$ равномерно no $u \in P$. 
ДоКАЗАТЕЛЬСтво. В силу свойства непрерьвности геометрической разности (см. $\left[5\right.$, c. 31]) для любого $\varepsilon>0$ существует $\delta=r_{2} \varepsilon /\left(2 r_{1}\right)$ такое, что для любого $u \in P$ выполняются включения

$$
H_{1}\left(\omega_{0}, u\right) \subset\left(H_{1}\left(\omega_{0}, u\right) \stackrel{*}{\mathbb{B}_{\delta}}\right)+\mathbb{B}_{\varepsilon}, \quad H_{2}\left(\omega_{0}, u\right) \subset\left(H_{2}\left(\omega_{0}, u\right) \stackrel{*}{\mathbb{B}_{\delta}}\right)+\mathbb{B}_{\varepsilon} .
$$

Далее существует $\eta>0$ такое, что для любого $\omega \in \Omega,\left|\omega-\omega_{0}\right|_{\mathbb{E}}<\eta$, и для любого $u \in P$ выполнены включения

$$
G_{0}(\omega, u) \subset G_{0}\left(\omega_{0}, u\right)+\mathbb{B}_{\delta / 2}, G_{1}\left(\omega_{0}\right) \subset G_{1}(\omega)+\mathbb{B}_{\delta / 2}, G_{2}\left(\omega_{0}\right) \subset G_{2}(\omega)+\mathbb{B}_{\delta}
$$

Следовательно, получаем

$H_{1}\left(\omega_{0}, u\right) \stackrel{*}{\mathbb{B}_{\delta}}=\left(G_{1}\left(\omega_{0}\right) \stackrel{*}{*} \mathbb{B}_{\delta / 2}\right) \stackrel{*}{*}\left(G_{0}\left(\omega_{0}, u\right)+\mathbb{B}_{\delta / 2}\right) \subset G_{1}(\omega) \stackrel{*}{*} G_{0}(\omega, u)=H_{1}(\omega, u)$.

Отсюда находим, что

$$
H_{2}\left(\omega_{0}, u\right) \stackrel{*}{*} \mathbb{B}_{\delta}=\left(H_{1}\left(\omega_{0}, u\right) \stackrel{*}{*} \mathbb{B}_{\delta}\right) \cap\left(G_{2}\left(\omega_{0}\right) * \mathbb{B}_{\delta}\right) \subset H_{1}(\omega, u) \cap G_{2}(\omega)=H_{2}(\omega, u) .
$$

Окончательно получаем, что

$$
H_{1}\left(\omega_{0}, u\right) \subset H_{1}(\omega, u)+\mathbb{B}_{\varepsilon}, \quad H_{2}\left(\omega_{0}, u\right) \subset H_{2}(\omega, u)+\mathbb{B}_{\varepsilon}
$$

для любого $\omega \in \Omega,\left|\omega-\omega_{0}\right|_{\mathbb{E}}<\eta$, и для любого $u \in P$. Лемма доказана.

ЛЕмма 5. Пусть $\mathbb{E}$ - конечномерное нормированное пространство с нормой | $\left.\right|_{\mathbb{E}}$. Пусть $\Omega-$ подмножество $\mathbb{E}$. Пусть многозначное отображсение $G: \Omega \times P$ $\rightarrow \mathscr{K}$ является полунепрерывным снизу в точке $\omega_{0} \in \Omega$ равномерно по и $\in P$. Пусть многозначное отображсение $H: \Omega \rightarrow \mathscr{K}$ таково, что

$$
H(\omega)=\operatorname{co} \bigcup_{u \in P} G(\omega, u)
$$

для любого $\omega \in \Omega$. Тогда $H$ полунепрерывно снизу в точке $\omega_{0} \in \Omega$.

ДокАЗАТЕльство. Так как $G$ является полунепрерьвньм снизу в точке $\omega_{0} \in \Omega$ равномерно по $u \in P$, то для любого $\varepsilon>0$ существует $\delta>0$ такое, что для любого $\omega \in \Omega$, $\left|\omega-\omega_{0}\right| \mathbb{E}<\eta$, и для любого $u \in P$ вьполнено включение

$$
G\left(\omega_{0}, u\right) \subset G(\omega, u)+\mathbb{B}_{\varepsilon} \subset H(\omega)+\mathbb{B}_{\varepsilon} .
$$

Следовательно, в силу произвольности $u \in P$ и выпуклости значений $H$ получаем

$$
H\left(\omega_{0}\right) \subset H(\omega)+\mathbb{B}_{\varepsilon} .
$$

Лемма доказана.

Лемма 6. Пусть выполнены условия теоремы 4. Пусть натуральное $K>T / \delta_{0}$. Пусть $\Omega_{K}$ - мнохсество, состоящее из всех разбиений $\omega=\left\{t_{k}\right\}_{k=0}^{K}$ отрезка $[0, T]$ мелкости $|\omega|<\delta_{0} ;$ при этом $\Omega_{K}$ рассматривается как подмножество нормированного пространства $\mathbb{R}^{K+1}$. Тогда многозначное отображсение $M_{0}: \Omega_{K} \rightarrow \mathscr{K}$ является полунепрерывным снизу в $\Omega_{K}$. 
ДоКАЗАТЕЛЬСТВо. По лемме 4 для любого $\omega \in \Omega_{K}$ и для любого $k \in \overline{0, K}$ вьполнено включение

$$
M_{k}(\omega) \subset(\|M\|+r T) \mathbb{B}_{1} .
$$

Поэтому существует достаточно большое $\rho>0$ такое, что замена множества $R$ на множество $R \cap \mathbb{B}_{\rho}$ не изменит значений $M_{k}(\omega)$ для любого $\omega \in \Omega_{K}$. Поэтому далее без ограничения общности будем считать, что $R$ является вьпуклым компактом в $\mathbb{R}^{n}$.

Многозначное отображение $\omega \mapsto M_{K}(\omega)=M$ является полунепрерывным снизу на $\Omega_{K}$. Предположим, рассуждая по индукции, что для $k \in \overline{0, K-1}$ многозначное отображение $\omega \mapsto M_{k+1}(\omega)$ является полунепрерывным снизу на $\Omega_{K}$. Многозначное отображение $\omega \mapsto R * \mathbb{B}_{r} \Delta t_{k}$ является полунепрерывным снизу на $\Omega_{K}$ в силу условия (6) и следствия $3\left[5\right.$, с. 34]. Многозначное отображение $(\omega, u) \mapsto F(u) \Delta t_{k}$ является полунепрерьвньм снизу в $\Omega_{K}$ равномерно по $u \in P$, так как $F(u) \subset \mathbb{B}_{r}$ для всех $u \in P$. Следовательно, многозначное отображение $(\omega, u) \mapsto \widehat{M}_{k}(\omega, u)$ является полунепрерьвным снизу в $\Omega_{K}$ равномерно по $u \in P$ в силу леммы 4 (условия леммы вьполнены для $\left.r_{1}=\|M\|+r T, r_{2}=r_{0}\right)$. Тогда в силу леммы 5 многозначное отображение $\omega \mapsto M_{k}(\omega)$ является полунепрерывным снизу в $\Omega_{K}$. Следовательно, по индукции доказана полунепрерьвность многозначного отображения $\omega \mapsto M_{0}(\omega)$ в $\Omega_{K}$. Лемма доказана.

ДоКАЗАТЕЛЬСТво ТЕОРЕМЫ 4. Покажем, что для любого $\varepsilon>0$ существует разбиение $\omega_{\varepsilon}$ отрезка $[0, T]$ мелкости $\left|\omega_{\varepsilon}\right|<\delta_{0}$ такое, что

$$
\widetilde{M} \subset M_{0}\left(\omega_{\varepsilon}\right)+\mathbb{B}_{\varepsilon} .
$$

Предположим, рассуждая от противного, что существует $\varepsilon_{0}>0$ такое, что для любого разбиения $\omega$ отрезка $[0, T]$ мелкости $|\omega|<\delta_{0}$ существует вектор $z(\omega) \in \widetilde{M}$ такой, что $z(\omega) \notin M_{0}(\omega)+\mathbb{B}_{\varepsilon_{0}}$. Рассмотрим последовательность разбиений $\omega_{m},\left|\omega_{m}\right| \rightarrow 0$ при $m \rightarrow \infty$. Пусть $z_{m}=z\left(\omega_{m}\right)$. Так как $\widetilde{M}-$ компакт, то, переходя, если нужно, к подпоследовательности, будем считать, что $z_{m} \rightarrow \tilde{z} \in \widetilde{M}$ при $m \rightarrow \infty$. По определению $\widetilde{M}$ существует разбиение $\tilde{\omega} \in \Omega_{\tilde{K}}$ такое, что $\tilde{z} \in M_{0}(\tilde{\omega})+(1 / 4) \mathbb{B}_{\varepsilon_{0}}$. По теореме 3 можно считать, что $|\tilde{\omega}|<\delta_{0}, \widetilde{K}>T / \delta_{0}$. Так как многозначное отображение $M_{0}: \Omega_{\widetilde{K}} \rightarrow \mathscr{K}$ полунепрерьвно снизу в $\widetilde{\omega}$ по лемме 6 и вьполнено условие $\left|\omega_{m}\right| \rightarrow 0$ при $m \rightarrow \infty$, то существует номер $\widetilde{m}$ такой, что для всех $m>\widetilde{m}$ найдется $\widetilde{\omega}_{m} \in \Omega_{\widetilde{K}}, \widetilde{\omega}_{m} \prec \omega_{m}$, для которого вьполнено включение

$$
M_{0}(\widetilde{\omega}) \subset M_{0}\left(\widetilde{\omega}_{m}\right)+\frac{1}{4} \mathbb{B}_{\varepsilon_{0}} \subset M_{0}\left(\omega_{m}\right)+\frac{1}{4} \mathbb{B}_{\varepsilon_{0}} .
$$

Последнее включение справедливо в силу теоремы 3 . Таким образом, $\tilde{z} \in M_{0}\left(\omega_{m}\right)+$ $(1 / 2) \mathbb{B}_{\varepsilon_{0}}$ для всех $m>\widetilde{m}$. С другой стороны, $z_{m} \notin M_{0}\left(\omega_{m}\right)+\mathbb{B}_{\varepsilon_{0}}$ для всех $m$. Так как $z_{m} \rightarrow \tilde{z}$ при $m \rightarrow \infty$, то существует номер $\widehat{m}$ такой, что для всех $m>\widehat{m}$ выполнено включение $\tilde{z} \in z_{m}+(1 / 2) \mathbb{B}_{\varepsilon_{0}}$. Следовательно, $\tilde{z} \notin M_{0}\left(\omega_{m}\right)+(1 / 2) \mathbb{B}_{\varepsilon_{0}}$ для всех $m>\widehat{m}$. Получили противоречие.

Итак, в нашем распоряжении для любого $\varepsilon>0$ существует разбиение $\omega_{\varepsilon}=\left\{t_{k, \varepsilon}\right\}_{k=0}^{K_{\varepsilon}}$ мелкости $\left|\omega_{\varepsilon}\right|<\delta_{0}$ такое, что выполнено включение (8). Определим число

$$
\delta(\varepsilon)=\min \left\{t_{k+1, \varepsilon}-t_{k, \varepsilon}: k \in \overline{0, K_{\varepsilon}-1}\right\} .
$$

Так как многозначное отображение $M_{0}: \Omega_{K_{\varepsilon}} \rightarrow \mathscr{K}$ является полунепрерывным снизу в $\omega_{\varepsilon}$ по лемме 6 , то существует $\gamma(\varepsilon)>0$ такое, что для любого разбиения $\omega=\left\{t_{k}\right\}_{k=0}^{K_{\varepsilon}} \in$ $\Omega_{K_{\varepsilon}}$ такого, что $\left|t_{k}-t_{k, \varepsilon}\right|<\gamma(\varepsilon)$ для всех $k \in \overline{0, K_{\varepsilon}}$, вьполнено включение

$$
M_{0}\left(\omega_{\varepsilon}\right) \subset M_{0}(\omega)+\mathbb{B}_{\varepsilon} .
$$


Рассмотрим произвольное разбиение $\omega$ отрезка $[0, T]$ мелкости $|\omega|<\min \{\delta(\varepsilon), \gamma(\varepsilon)\}$. Тогда существует разбиение $\widetilde{\omega}=\left\{\tilde{t}_{k}\right\}_{k=0}^{K_{\varepsilon}} \in \Omega_{K_{\varepsilon}}$, причем $\widetilde{\omega} \prec \omega$ и $\left|\tilde{t}_{k}-t_{k, \varepsilon}\right|<\gamma(\varepsilon)$ для всех $k \in \overline{0, K_{\varepsilon}}$. Следовательно, получаем

$$
M_{0}\left(\omega_{\varepsilon}\right) \subset M_{0}(\widetilde{\omega})+\mathbb{B}_{\varepsilon} \subset M_{0}(\omega)+\mathbb{B}_{\varepsilon} .
$$

Последнее включение справедливо в силу теоремы 3. Таким образом, справедливы включения

$$
M_{0}(\omega) \subset \widehat{M} \subset M_{0}(\omega)+2 \mathbb{B}_{\varepsilon},
$$

т.е. справедливо неравенство $h\left(M_{0}(\omega), \widetilde{M}\right) \leqslant 2 \varepsilon$ для любого разбиения $\omega$ отрезка $[0, T]$ мелкости $|\omega|<\min \{\delta(\varepsilon), \gamma(\varepsilon)\}$. Теорема доказана.

Заметим, что соотношение (6) в условии теоремы 4 выполнено для всех достаточно мелких разбиений отрезка $[0, T]$, если оно выполняется хотя бы для одного разбиения этого отрезка. Таким образом, справедлива следующая

ТЕОРема 5. Пусть существует $r_{0}>0$ и разбиение $\omega_{0}=\left\{t_{k}^{(0)}\right\}_{k=0}^{K_{0}}$ отрезка $[0, T]$ такие, что

$$
\widehat{M}_{k}\left(\omega_{0}, u\right) \stackrel{*}{\mathbb{B}_{2 r_{0}}} \neq \varnothing
$$

для всех $u \in P, k \in \overline{0, K_{0}-1}$. Тогда существует $\delta_{0}>0$ такое, ито для любого разбиения $\omega=\left\{t_{k}\right\}_{k=0}^{K}$ отрезка $[0, T]$ мелкости $|\omega|<\delta_{0}$ и любого и $\in P$ выполнено (6).

ДокАЗАТЕЛЬСТво. Определим множество

$$
\Omega_{0}=\left\{\omega=\left\{t_{k}\right\}_{k=0}^{K_{0}}: 0=t_{0}<t_{1}<\cdots<t_{K_{0}}=T\right\} .
$$

Рассматриваем $\Omega_{0}$ как подмножество нормированного пространства $\mathbb{R}^{K_{0}+1}$. Так же, как и в доказательстве леммы 6 , без ограничения обшности считаем, что $R$ является выпукльм компактом в $\mathbb{R}^{n}$. Докажем, что для любого $k \in \overline{0, K_{0}-1}$ многозначные отображения $\widehat{M}_{k}: \Omega_{0} \times P \rightarrow \mathscr{K}$ являются полунепрерывными снизу в точке $\omega_{0}$ равномерно по $u \in P$. Предположим, рассуждая по индукции, что многозначное отображение $M_{k+1}: \Omega_{0} \rightarrow \mathscr{K}$ полунепрерьвно снизу в точке $\omega_{0}$ (для $k=K_{0}-1$ это верно, так как по определению $M_{K_{0}}(\omega)=M$ для любого $\left.\omega \in \Omega_{0}\right)$. Тогда в силу леммы 4 многозначное отображение $\widehat{M}_{k}$ полунепрерывно снизу в точке $\omega_{0}$ равномерно по $u \in P$. Далее, в силу леммы 5 многозначное отображение $M_{k}$ полунепрерьвно снизу в точке $\omega_{0}$. Таким образом, по индукции доказана полунепрерывность снизу многозначных отображений $\widehat{M}_{k}$ в точке $\omega_{0}$ равномерно по $u \in P$. Следовательно, существует $\gamma_{0}>0$ такое, что для любого разбиения $\omega_{1}=\left\{t_{k}^{(1)}\right\}_{k=0}^{K_{0}}$ такого, что $\left|t_{k}^{(1)}-t_{k}^{(0)}\right|<\gamma_{0}$, где $k \in \overline{0, K_{0}}$, и любого вектора $u \in P$ выполнено включение

$$
\widehat{M}_{k}\left(\omega_{0}, u\right) \subset \widehat{M}_{k}\left(\omega_{1}, u\right)+\mathbb{B}_{r_{0}} \quad \forall k \in \overline{0, K_{0}-1} .
$$

В силу соотношения (9) получаем

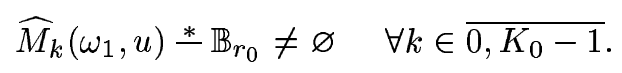

Пусть $\eta_{0}=\min \left\{t_{k}^{(0)}-t_{k-1}^{(0)}: k \in \overline{1, K_{0}}\right\}$. Определим $\delta_{0}=\min \left\{\gamma_{0}, \eta_{0}\right\}$. Рассмотрим произвольное разбиение $\omega=\left\{t_{k}\right\}_{k=0}^{K}$ отрезка $[0, T]$ мелкости $|\omega|<\delta_{0}$. Тогда сушествует разбиение $\omega_{1}=\left\{t_{k}^{(1)}\right\}_{k=0}^{K_{0}}$ такое, что $\omega_{1} \prec \omega$ и $\left|t_{k}^{(1)}-t_{k}^{(0)}\right|<\gamma_{0}$ для всех $k \in \overline{0, K_{0}}$. 
В силу (10), для любого $k \in \overline{0, K_{0}-1}$ и любого $u \in P$ сушествует вектор $a_{k}(u) \in \mathbb{R}^{n}$ такой, что справедливо включение

$$
a_{k}(u)+\mathbb{B}_{r_{0}} \subset \widehat{M}_{k}\left(\omega_{1}, u\right) .
$$

В силу соотношения $\omega_{1} \prec \omega$ существуют номера $\left\{s_{k}\right\}_{k=0}^{K_{0}}$ такие, что $0=s_{0}<s_{1}<\cdots$ $<s_{K_{0}}=K$ и $t_{s_{k}}=t_{k}^{(1)}$ для всех $k \in \overline{0, K_{0}}$. В силу (11) для любого $k \in \overline{0, K_{0}-1}$ и произвольного вектора $u \in P$ получаем

$$
\begin{gathered}
a_{k}(u)+\mathbb{B}_{r_{0}}+\left(t_{s_{k+1}}-t_{s_{k}}\right) F(u) \subset M_{s_{k+1}}(\omega), \\
a_{k}(u)+\mathbb{B}_{r_{0}} \subset R \stackrel{*}{*}\left(t_{s_{k+1}}-t_{s_{k}}\right) \mathbb{B}_{r} .
\end{gathered}
$$

Включение (12) имеет место также в силу включения $M_{k+1}\left(\omega_{1}\right) \subset M_{s_{k+1}}(\omega)$, которое справедливо по теореме 3 . В силу (13) для любого $j \in \overline{s_{k}, s_{k+1}}$ и произвольного $u \in P$ выполнено включение

$$
a_{k}(u)+\mathbb{B}_{r_{0}}+\left(t_{j}-t_{s_{k}}\right) F(u) \subset R \stackrel{*}{*}\left(t_{s_{k+1}}-t_{j}\right) \mathbb{B}_{r} .
$$

Зафиксируем номер $i \in \overline{s_{k}, s_{k+1}-1}$. Для завершения доказательства теоремы требуется для любого $u \in P$ доказать неравенство

$$
\widehat{M}_{i}(\omega, u) \stackrel{*}{\mathbb{B}_{r_{0}}} \neq \varnothing .
$$

Докажем его, рассуждая по индукции. Пусть для некоторого номера $j \in \overline{i+1, s_{k+1}}$ и для любого $u \in P$ справедливо включение

$$
a_{k}(u)+\mathbb{B}_{r_{0}}+\left(t_{j}-t_{s_{k}}\right) F(u) \subset M_{j}(\omega) .
$$

Для $j=s_{k+1}$ это включение имеет место в силу (12). Следовательно, вьполнены включения

$$
\begin{aligned}
& a_{k}(u)+\mathbb{B}_{r_{0}}+\left(t_{j-1}-t_{s_{k}}\right) F(u) \subset M_{j}(\omega) \stackrel{*}{*} F(u) \Delta t_{j-1}, \\
& a_{k}(u)+\mathbb{B}_{r_{0}}+\left(t_{j-1}-t_{s_{k}}\right) F(u) \subset R \stackrel{*}{\mathbb{B}_{r} \Delta t_{j-1} .}
\end{aligned}
$$

Последнее включение вьполнено в силу (14) и соотношения $\left(t_{s_{k+1}}-t_{j-1}\right) \mathbb{B}_{r} \supset \mathbb{B}_{r} \Delta t_{j-1}$. Следовательно, для любого $u \in P$ получаем

$$
a_{k}(u)+\mathbb{B}_{r_{0}}+\left(t_{j-1}-t_{s_{k}}\right) F(u) \subset \widehat{M}_{j-1}(\omega, u) \subset M_{j-1}(\omega) .
$$

Таким образом,

$$
\widehat{M}_{j-1}(\omega, u) \stackrel{*}{ } \mathbb{B}_{r_{0}} \neq \varnothing,
$$

т.е. по индукции соотношение (15) установлено. Следовательно, для любого разбиения $\omega=\left\{t_{k}\right\}_{k=0}^{K}$ отрезка $[0, T]$ мелкости $|\omega|<\delta_{0}$ и любого вектора $u \in P$ вьполнено (6). Теорема доказана.

\section{СПИСОК ЦИТИРОВАННОЙ ЛИТЕРАТУРЫ}

[1] Айзекс Р Дифференциальные игры. М.: Мир, 1967.

[2] Красовский Н. Н. Управление динамической системой. Задача о минимуме гарантированного результата. М.: Наука, 1985.

[3] Понтрягин Л.С. Линейные дифференциальные игры преследования // Матем. сб. 1980. T. 112. № 3. C. $307-330$.

[4] Пшеничный Б. Н. Выпуклый анализ и экстремальные задачи. М.: Наука, 1980.

[5] Половинкин Е.С. Элементы теории многозначных отображений. Учебное пособие. М.: МФТИ, 1982. 